\title{
Erste Desmopressin-Schmelztablette bei Enuresis nocturna
}

— Die seit 1. Juni in Deutschland erhältliche Schmelztablette zur Behandlung der Enuresis nocturna "Minirin ${ }^{\oplus} 60$ I 120 I 240 Mikrogramm Lyophilisat zum Einnehmen“ ist die Weiterentwicklung des Klassikers Minirin ${ }^{\circledast}$ (Wirkstoff bei beiden: Desmopressin).

In der Behandlung der primären Enuresis nocturna (PEN) steht ein symptomorientiertes und verhaltenstherapeutisches Vorgehen im Vordergrund: Techniken zum Verhaltenstraining und zur Selbstbeobachtung

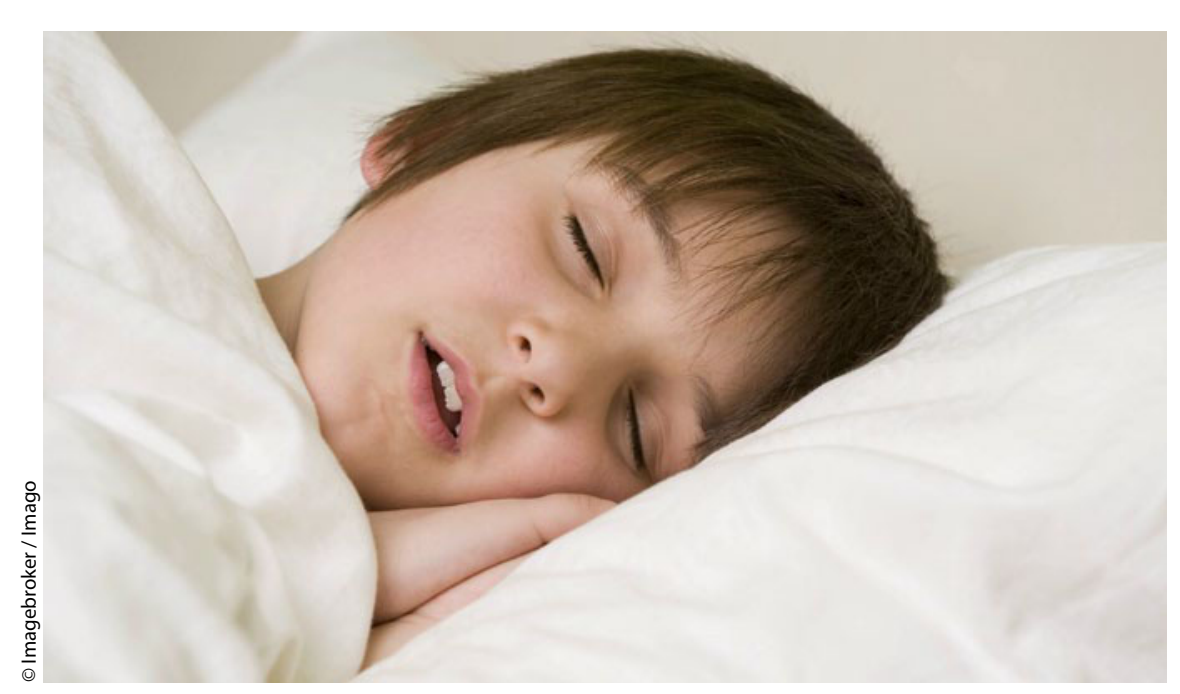

Endlich trockene Nächte? Rund 10\% der Schulanfänger leiden an Enuresis nocturna.

(Urotherapie) werden zum Beispiel durch eine medikamentöse Behandlung ergänzt. Der bei PEN bewährte Wirkstoff Desmopressin wirkt wie das körpereigene Antidiuretische Hormon (ADH), das die nächtliche Urinproduktion reduziert. Bei bettnässenden Kindern wird ADH nachts unzureichend ausgeschüttet. Desmopressin wirkt dem entgegen. Wird bei Therapieende nach einem strukturierten Therapieschema ausgeschlichen, besteht für die Patienten eine signifikant höhere Wahrscheinlichkeit, trocken zu bleiben, als bei Patienten, bei denen die Therapie abrupt abgebrochen wird.

Die Minirin ${ }^{\circledR}$-Schmelztablette kommt mit einer deutlich geringeren Wirkstoffbelastung aus - bei gleicher Wirksamkeit und Verträglichkeit wie die Minirin ${ }^{\circledast}$-Tablette: $120 \mu \mathrm{g}$ statt $200 \mu \mathrm{g}$ für die Anfangsdosis, $240 \mu \mathrm{g}$ statt $400 \mu \mathrm{g}$ für die Titrationsdosis. Die Urinkonzentration beginnt 30 Minuten nach der Einnahme.

Als erste und einzige Schmelztablette in der Enuresis-Therapie wird Minirin ${ }^{\oplus}$-Lyophilisat zum Einnehmen von Kindern bevorzugt, wie eine randomisierte Open-Label-, Cross-Over Studie belegt. Bei $60 \%$ der unter 12-jährigen Patienten konnte die Präferenz für das Lyophilisat signifikant nachgewiesen werden $(p=0,009)$. Die Untersuchung zeigte zusätzlich eine hohe Compliance bei der Einnahme der Schmelztablette. Der Vorteil der Schmelztablette: Sie wird einfach unter die Zunge gelegt und löst sich dort sofort auf - es wird keine Flüssigkeit zur Einnahme benötigt. Die Schmelztablette ist neben Enuresis auch in den Indikationen Diabetes insipidus, Nykturie und Polyurie zugelassen.

Nach Informationen von Ferring, Kiel

\section{Hyaluronan: GAG-Schicht-Bestandteil mit erwiesener Langzeitwirkung}

- Eine Studie macht aktuell in Fachkreisen auf sich aufmerksam: Prof. Claus R. Riedl und Kollegen haben kürzlich die Ergebnisse einer Langzeitstudie veröffentlicht, mit der sie die Wirksamkeit von Hyaluronan (Cystistat ${ }^{\oplus}$ ) bei der Interstitiellen Zystitis über einen Zeitraum von fünf Jahren untersuchten. Die Ergebnisse sind unter dem Titel „Long-term results of intravesical hyaluronan therapy in bladder pain syndrome/interstitial cystitis" online im International Urogynecology Journal erschienen.

Die kurzfristige Wirkung der intravesikalen Instillation von Hyaluronan bei Interstitieller Zystitis (IC) und chronischem Blasenschmerzsyndrom (BPS) konnte in vielen Studien gezeigt werden. Sie basiert darauf, dass Hyaluronan die Heilung von Defekten in der Glycosaminoglycan-Schicht (GAGSchicht) fördert, sodass ein Eindringen pa- thogener Keime in das Blasen- und Harnröhrenepithel verhindert wird. Außerdem wurden antientzündliche Mechanismen nachgewiesen.

Im Rahmen der aktuellen Studie wurden 70 Patientinnen mit BPS/IC, die zwischen 2001 und 2003 mit Hyaluronan behandelt worden waren, Fragen zu ihrer aktuellen gesundheitlichen Situation beantworten. Die Auswertung dieser Befragung liefert die bisher noch fehlenden Daten zur langfristigen Wirksamkeit der Hyaluronantherapie: Bei den 48 Patientinnen, welche die Fragen beantwortet haben, lag der VAS-Score (0-10) für blasenspezifische Symptome bei 2,14 . Fünf Jahre zuvor, also vor Beginn der Therapie, hatte er bei 8,15 gelegen. $50 \%$ der Patientinnen waren zum Zeitpunkt der Befragung noch immer symptomfrei. $25 \%$ der Patientinnen waren symptom- frei, nachdem sie nach wiederkehrenden Beschwerden einen weiteren HyaluronanBehandlungszyklus erhalten hatten; weitere $16,7 \%$ waren symptomfrei, nachdem sie eine Hyaluronantherapie in Verbindung mit der oralen Gabe von PPS (Pentosanpolysulfat) erhalten hatten. Bei 8,3\% war kein Ansprechen auf die Therapie festzustellen. Die Ergebnisse der Studie legen nahe, dass die dauerhafte Heilung der Krankheit BPS/ IC allein durch die Behandlung mit Hyaluronan bei einem großen Teil der Betroffenen möglich ist.

Nach Informationen von

Uromed, Oststeinbek 